\title{
Efficacy Study With Post-Exposure Radiomitigation
}

National Cancer Institute

\section{Source}

National Cancer Institute. Efficacy Study With Post-Exposure Radiomitigation. NCI

Thesaurus. Code C158358.

A study that assesses the efficacy of a radiomitigator given after exposure to the challenge agent(s) but before the manifestation of the disease or condition. 